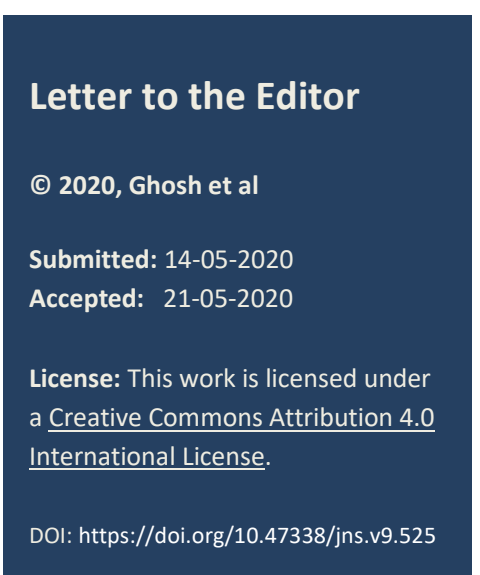

\section{Primary iliopsoas abscess in a neonate: A diagnostic dilemma}

\author{
Soumyodhriti Ghosh, ${ }^{1 *}$ Abhijit Kundu, ${ }^{2}$ Padma Lochan Rath, ${ }^{3}$ Abhishek Kumar, ${ }^{4}$ \\ 1 Consultant Pediatric Surgeon, Abhishek Child Care Hospital, Jamshedpur, Jharkhand \\ 2 Consultant General Surgeon, Abhishek Child Care Hospital, Jamshedpur, Jharkhand \\ 3 Consultant Anesthesiologist, Abhishek Child Care Hospital, Jamshedpur, Jharkhand \\ 4 Consultant Pediatrician, Abhishek Child Care Hospital, Jamshedpur, Jharkhand
}

Correspondence*: Soumyodhriti Ghosh, Pediatric Surgery Unit, Sixth floor, Abhishek Child Care Hospital, Jharkhand, India. E-mail: drsghosh.surg85@gmail.com

\section{Dear Editor}

Iliopsoas abscess is uncommon in children and rare still in neonates.[1,2] A 10-day-old female neonate presented to the pediatric surgery emergency with high grade fever and swelling of the right upper thigh. The neonate was sick looking, dehydrated, and febrile. The limb swelling was confined to the right medial upper thigh. On careful palpation, a fluctuation bulge could also be appreciated over the right flank. Both the swellings appeared tender to touch. Additionally, the baby had restricted movement of the right lower limb and cried on manipulation of the limb. She was started on intravenous ampicillin and cloxacillin antibiotics. Laboratory investigations revealed neutrophilic leukocytosis and elevated C-reactive protein. Blood culture was sterile.

Hip joint radiograph ruled out septic arthritis, which was our provisional diagnosis. Spine was also unremarkable. Ultrasound of both swellings depicted it right sided iliopsoas abscess extending from the iliac crest to the medial thigh. Percutaneous drainage attempted but failed due to loculated collection and viscosity of pus. The baby underwent open, extra peritoneal drainage of the collection. Pus microscopy showed scant gram-positive cocci; pus culture was sterile. Systemic antibiotics were continued up to two weeks. The neonate responded well with clinical betterment and normalization of blood counts. Postoperative period was uneventful. Follow-up at six weeks showed no fresh collections with normal limb movements.

Iliopsoas abscess is very rare in neonatal age with less than twenty cases reported till date.[1]The common presentations are swelling and restricted mobility of limb along with fever as noted in the index case. Unilateral lesions are common, though bilateral cases have also been reported.[2]
The etiology of primary iliopsoas abscess is unknown with hematogenous spread from occult site commonly implicated.[1,4] Psoas abscess may also be secondary to septic arthritic collections, osteomyelitis of femur, spread from abscess at vaccine site or traumatic cellulitis. None could be found, as an etiology, in the index case. The common causative organisms are Staphylococcus aureus followed by Klebsiella and streptococcus. Ultrasound is the investigation of choice.

CT scan may complement by showing the extent of collection and planning drainage. However, CT scan is often not performed due to the acute presentation and radiation hazard to the neonate. Similarly, in the index case, surgical decision making was done based on ultrasonographic findings. Simple medical management is not always sufficient and pus aspiration, or incision and drainage of abscess may be needed.[1,5,6] The prognosis stands good with an early recovery in the absence of predisposing factors like immune compromise status, septic arthritis, spondylodiscitis of spine or MRSA infection. $[1,5]$

However, a high index of suspicion is warranted to reach the diagnosis in case of primary neonatal iliopsoas abscess. Delay in diagnosis and inadequate pus drainage may lead to sepsis, mortality and sequelae of damage to the joints. $[5,6]$

Acknowledgements: Nil

Conflict of Interest: None declared

Source of Support: Nil

Consent to Publication: No clinical figure is used in this manuscript.

Author Contributions: Author(s) declared to fulfil authorship criteria as devised by ICMJE and approved the final version. 


\section{REFERENCES}

1. Duminda HK, Lamahewage AK, Liyange A, Fernando DM. Iliopsoas abscess due to methicillin resistant staphylococcus aureus in a 26 day old neonate. Sri Lanka J Child Health. 2015; 44:167-8.

2. Schut JM, Meradji M, Oranje AP, Bergmeijer JH, Schuller JL. Double-sided psoas abscess in a young infant: sonographic and radiographic findings. Pediatric radiology. $1988 ; 18: 176-7$.
3. Ishibashi H, Oshio T, Sogami T, Nii A, Mori H, Yada K, et al. Iliopsoas abscess in an infant. J Med Inv. 2014; 61:213-6.

4. Patel R, Pimpalwar A, Hutton K. Primary neonatal iliopsoas abscess. J Pediatr Surg Case Rep. 2013; 1:113.

5. Mondal R, Sarkar S. Spondylodiscitis with primary psoas abscess in a neonate. Ind Pediatr. 2012; 49:681.

6. Horiuchi A, Kameoka K, Kuwabara J, Watanabe Y, Kawakami S, Tauchi $\mathrm{H}$, et al. Neonatal iliopsoas abscess. Pediatr Int. 2012; 54:712-4. 